\title{
Kawasaki Disease and Labyrinthitis: An Underdiagnosed Complication
}

\author{
Kyu Yeun Kim ${ }^{1}$, Ki Hwan Kim¹, Yoon Ah Park ${ }^{2}$, and Young Joon Seo ${ }^{2}$ \\ 'Department of Pediatrics, Yonsei University College of Medicine, Seoul, \\ ${ }^{2}$ Department of Otorhinolaryngology, Yonsei University Wonju College of Medicine, Wonju, Korea
}

Received July 18, 2016

Revised August 17, 2016

Accepted August 17, 2016

Address for correspondence

Young Joon Seo, MD, PhD

Department of Otorhinolaryngology,

Yonsei University

Wonju College of Medicine,

20 Ilsan-ro, Wonju 26426, Korea

Tel $+82-33-741-0642$

Fax $+82-33-732-8287$

E-mail okas2000@yonsei.ac.kr
Sensorineural hearing loss (SNHL) that is seldom cited as a Kawasaki disease (KD) complication is known as an additional, potentially severe, and frequently irreversible sequel. Furthermore the vestibular functions of KD have been underestimated and it could be an important complication combined with SNHL in KD. We described a case that a 4 year-old boy who developed vestibular loss with SNHL has recovered successfully with a combined treatment.

J Audiol Otol 2017;21(1):53-56

KEY WORDS: Kawasaki disease · Labyrinthitis · Sensorineural hearing loss.

\section{Introduction}

Kawasaki disease (KD) is a self-limited, systemic vasculitis of unknown etiology that affects the small and mediumsized blood vessels of the body [1]. The diagnostic criteria for $\mathrm{KD}$ are outlined in Table 1 . Over $75 \%$ of cases occur in children less than 5 years of age, and the incidence is highly varied, ranging from 4 to 216 per 100,000 children less than 5 years of age worldwide. The most serious complication of the disease is coronary vasculitis, leading to abnormalities in the coronary arteries that affect $15 \%$ to $25 \%$ of untreated patients [2]. In addition to the cardiac complications, other abnormalities such as ataxia, facial paralysis, behavior disorders, and ocular complications can also be present. Sensorineural hearing loss (SNHL) is seldom cited as a KD complication yet is known as an additional, potentially severe, and frequently irreversible sequel. However, the vestibular effects of KD have been underestimated, and these could also be an important complication combined with SNHL in KD.

This is an Open Access article distributed under the terms of the Creative Commons Attribution Non-Commercial License (http://creativecommons.org/licenses/by-nc/4.0/) which permits unrestricted non-commercial use, distribution, and reproduction in any medium, provided the original work is properly cited.
Here we describe the case of a 4-year-old boy who developed vestibular loss in addition to SNHL and recovered successfully with a combined treatment.

\section{Case Report}

A 4-year-old boy with no relevant prenatal or neonatal background and no history of hearing impairment had adequate normal growth and normal development of vocabulary and language. He was admitted to our Pediatrics Department due to uncontrolled fever and sudden onset vertigo persisting for 4 days. On admission, he presented with bilateral non-exudative conjunctival injection, marked injected and fissured lips, polymorphous rash of the face and trunk, and cervical lymphadenopathy (right multiple palpable Lymph nodes $>2$ $\mathrm{cm}$ ), in addition to the fever (Fig. 1). He had moved back and forth from severe nausea and vomiting to loss of balance; however, visual acuity and brain MRI was normal.

Laboratory workup revealed leukocytosis $(19,000 / \mu \mathrm{L})$ with neutrophilia (79.5\%), and neither anemia nor thrombocytosis was detected. Renal function tests and liver enzymes were within the normal range. Upon performing a lumbar puncture, there were no signs of any infectious results for meningitis. Negative serological tests were obtained for 
toxoplasmosis, mononucleosis, rubella, and cytomegalovirus. An electrocardiogram was normal, and no echocardiogram abnormalities were observed, with results showing normal dimensions of the coronary arteries.

A clinical diagnosis of $\mathrm{KD}$ was established according to the American Heart Association criteria. Treatment with intravenous immunoglobulin was promptly initiated $(1.5 \mathrm{~g} / \mathrm{kg}$ in a single and continuous perfusion) in association with acetylsalicylic acid (ASA) (80 mg/kg/day). He became apyretic within 24 hours of treatment implementation, with progressive regression of the remaining clinical symptoms. He soon recovered from nausea and vertigo after the treatments and was discharged 5 days later, maintaining ASA with low-dose prophylaxis ( $5 \mathrm{mg} / \mathrm{kg} /$ day $)$.

Four days after discharge, his parents noticed a sudden absence of response to sound stimuli and brought him to the clinic of otorhinolaryngology. Assessment of hearing function was obtained through impedance audiometry, play audiometry, and auditory brainstem response (ABR) (Fig. 2). His impedance audiometry showed a type A curve bilaterally with no response of stapedial reflex at a sound of $100 \mathrm{~dB}$ HL. Play pure tone audiometry confirmed severe bilateral SNHL with right mixed hearing loss components (right ear, $75 \mathrm{~dB}$ with an air-bone gap of $20 \mathrm{~dB}$; left ear, $45 \mathrm{~dB}$ ). A V wave in the $A B R$ test was present until $75 \mathrm{~dB}$ stimuli on the right ear and $55 \mathrm{~dB}$ on the left ear. A caloric test by air for vestibular evaluation showed right complete canal paresis. After the exclusion of salicylate toxicity (therapeutic drug monitoring level $<0.3 \mu \mathrm{g} / \mathrm{mL}$ plasma), we thought that the hearing loss could possibly be due to the subacute phase after the acute phase of KD. Blood tests confirmed thrombocytosis during the second week of disease $(606,000 / \mathrm{mL}$, compared with $416,000 / \mathrm{mL}$ at the initial admission) as well as a progressive decrease of acute inflammatory response markers $(\mathrm{WBC}=89,400 / \mu \mathrm{L}$; Neutrophil percentage $=40.3 \%$ ). There were no visualizations of either anterior inferior cerebellar artery (AICA), the feeding vessel of the cochlea, in 3D-magnetic resonance angiography (MRA) (Fig. 3).

To stop the progression of KD we administered a corticosteroid for immunosuppression and added an anticoagulant
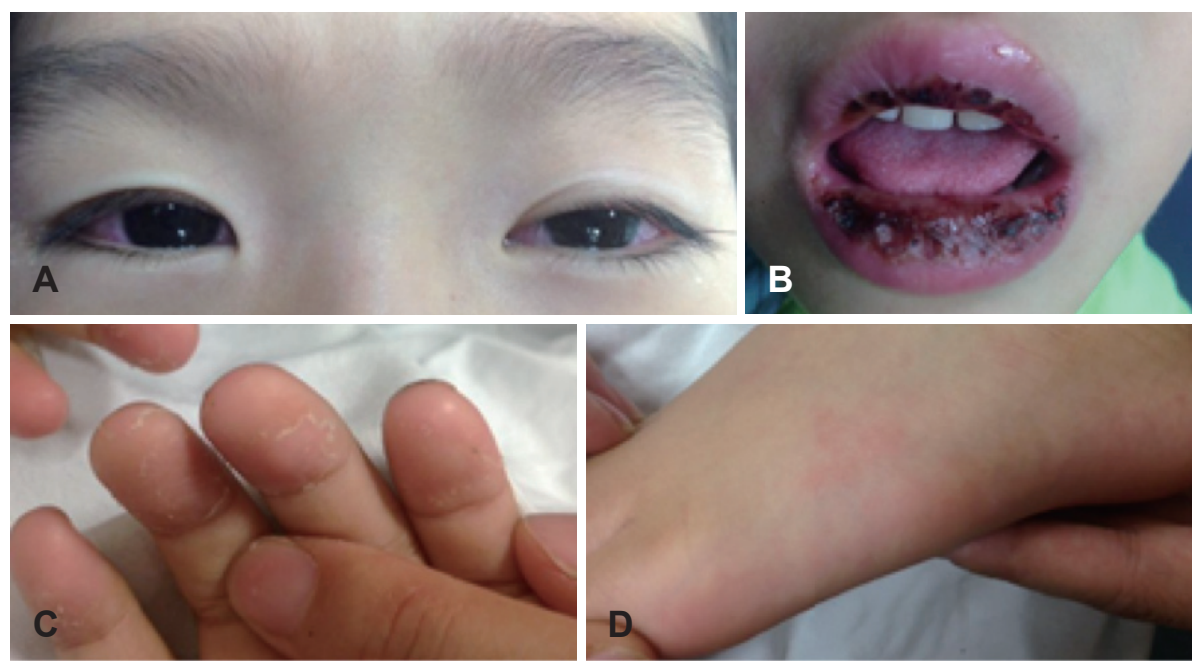

Fig. 1. Typical features of Kawasaki disease were shown. Bilateral nonexudative conjuctival injection (A), the marked injected and fissured lips (B), peeling of fingers (C) and solar erythema (D) was observed on his first visit.
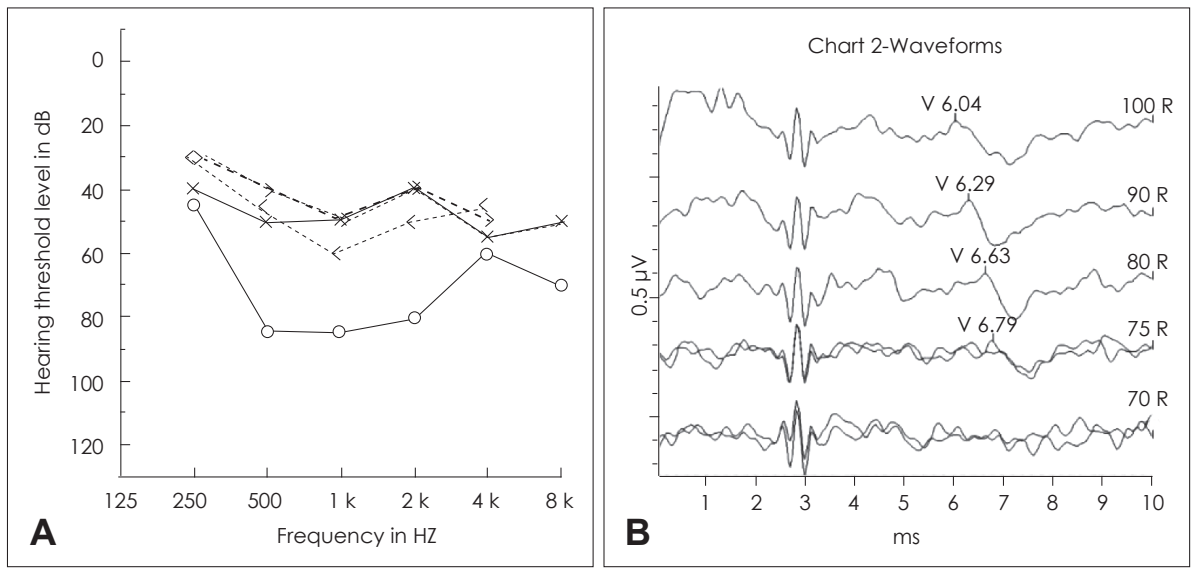

Fig. 2. Initial assessments of hearing function. A: The results of pure tone audiometry (PTA) showed right severe mixed hearing loss $(75 \mathrm{~dB})$ and left moderate sensorineural hearing loss $(45 \mathrm{~dB})$. Auditory brainstem response were consistent with PTA. B: $75 \mathrm{~dB}$ on the right ear. 
drug to interrupt the vascular insufficiency of the cochlea. Intravenous steroid pulse therapy (methylprednisolone sodium succinate, started at $200 \mathrm{mg}$ /day initially for 5 days and then tapered down) was initiated with Plavix (clopidogrel bisulfate, $18 \mathrm{mg} / \mathrm{kg}$ ), instead of ASA.

During follow-up, there was a significant improvement of auditory acuity in both ears ( 5 days after steroid therapy: right ear $=50 \mathrm{~dB}$, left ear $=45 \mathrm{~dB} ; 3$ weeks later: both $=25 \mathrm{~dB})($ Fig. 4). Steroid therapy was tapered off gradually and stopped after 3 weeks. The canal paresis of the right ear still remained unchanged. Tonal audiometry, performed 2 months after the diagnosis of KD, confirmed a normal hearing threshold. Cardiovascular assessment showed no evidence of coronary ectasia or aneurysm at follow-up.

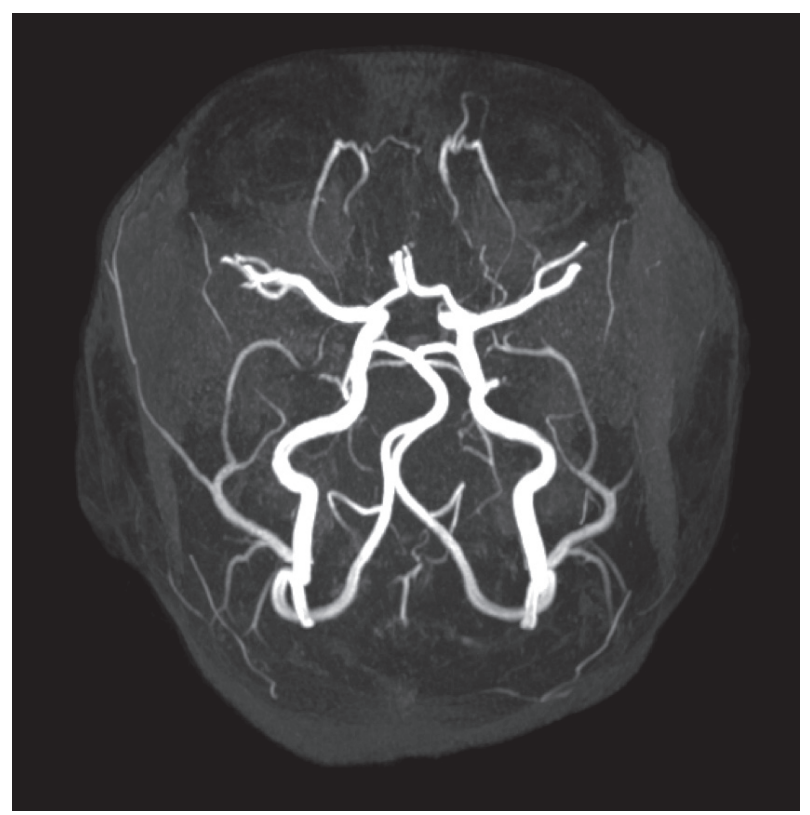

Fig. 3. There is no evidence of narrowing of both anterior inferior cerebellar arteries and feeding vessels of the cochlea, on the patient's 3D-magnetic resonance angiography.

\section{Discussion}

The major complications of KD are related to cardiac involvement, especially coronary vasculitis [2-4]. We observed another kind of severe complication in this case: SNHL and vestibular loss; which is not frequently reported in the literature $[5,6]$. The presence of hearing loss during the subacute phase of KD in children is very rare, especially when accompanied by canal paresis causing vestibular hypofunction. Moreover, the successful combined treatment restored the functions of the cochlea completely. Therefore, this report is important for assessing effective treatment in these patients.

Until now, the association of SNHL with KD seems to be underestimated, as hearing dysfunction can be discreet and transient in this disease, detectable only by audiometry or BERA in very young children who cannot express themselves well linguistically. Knott, et al. [5] found that 19 of 62 patients experienced SNHL with KD. Similarly, in the most recent and largest study of $115 \mathrm{KD}$ patients by Alves, et al., [7] $33 \%$ had SNHL during the acute and subacute phases of the disease. In the present case, the patient's parents failed to identify the hearing problem early after discharge and brought him to our clinic 4 days later, when he failed to correctly recognize words, even when hearing his own speech. Audiometry results then revealed that his right ear threshold was 75 $\mathrm{dB}$, and his left ear threshold was $40 \mathrm{~dB}$.

The pathogenesis of SNHL is unknown in patients with KD. The hearing loss may be associated with the aberrant inflammatory process, due to the intense immune activation observed in the acute phase [8,9]. This systemic inflammatory process can affect the membranes of the labyrinth and the osmotic balance in the fluid inside the compartments of the inner ear or in the cochlear vessels, thus leading to hearing loss [10]. Secondary abnormalities of the vasa nervorum and perineural vessels resulting in damage of the acoustic nerve could be other possible causes of hearing loss. The inflam-
Fig. 4. The results of pure tone audiometry at follow-up periods (A graph at 5 days after the treatment and $B$ graph at 3 weeks later). The improvement of more than $40 \mathrm{~dB}$ (on the right ear) was occurred after the treatment as well as on the left ear.
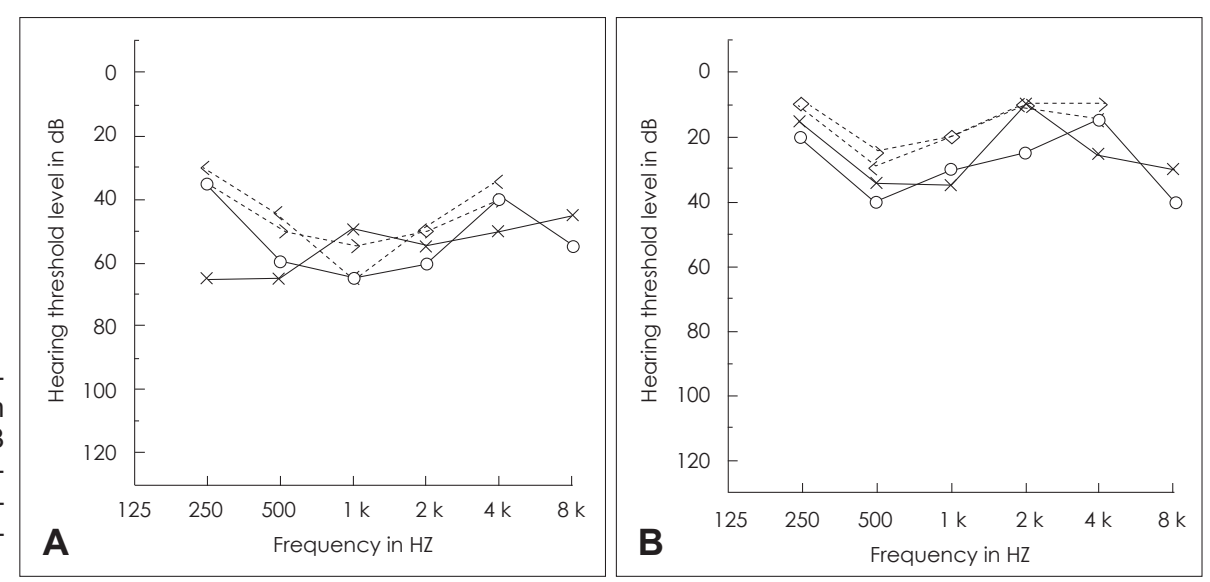
matory cells that infiltrate the vasa nervorum and epineural arteries lead to obstruction, axonal ischemia, and consequent nerve injury. The changed expression of metalloproteinases in the vessel walls can contribute to their subsequent destruction, which can participate directly in the injury caused by nerve ischemia [11]. In addition to this pathophysiology of $\mathrm{KD}$, thrombocytosis in this case indicated a possibility of ischemia injury owing to vascular insufficiency. The AICA, the feeding vessel of the cochlea and vestibule, was not visible on the images from 3D-MRA. However, we are uncertain as to whether the small vessels in both ears were obstructed or were too small to be visualized. The potential role of salicylate ototoxicity in the genesis of hearing loss in acute KD patients has been raised. Higher doses tend to cause more severe hearing loss, although a precise dose-response relationship cannot be demonstrated [12]. It is unlikely, however, that the SNHL experienced in this case was caused by ASA ototoxicity alone. The therapeutic drug monitoring level was very low $(<0.3 \mu \mathrm{g} / \mathrm{mL}$ plasma), and the patterns of hearing impairment differed from the patterns characteristic of salicylate ototoxicity, in which binaural flat or high-frequency deficits predominate. At the initial audiometry, there was an air bone gap that recovered within a few days. Although it might have been an error from the young child's play audiometry, we think that acute cochlear injury caused a stiffness of the ossicular chains, especially the stapes, due to a lateralization of oval window caused by endolymphatic hydrops [13].

$\mathrm{KD}$ can result in ataxia due to the involvement of the vestibular division of the acoustic nerve. Other cranial nerves may also be affected, and facial paralysis secondary to involvement of the seventh nerve has also been described [14]. Although this patient had initial symptoms of a vestibular deficit with severe nausea, no one was able to determine when the hearing loss started. The results of the caloric test indicated no response from the right ear canal, showing that dizziness and ataxia might have originated from a complete loss of vestibular function. Despite the fact that the symptoms of nausea and unbalance subsided due to a compensation of the contralateral side, the canal paresis still remained.

The early treatment initiated during the first week of hearing loss, including the use of high-dose systemic steroids combined with anticoagulants, is thought to possibly prevent the progression of hearing deficit. Complete remission within two months was obtained due to early awareness of the possibility of SNHL associated with $\mathrm{KD}$, which allowed for a timely intervention.

Children with KD may be referred to the otolaryngologist for a variety of signs and symptoms during their acute illness. Referral for evaluation of dizziness as well as hearing loss may occur long after signs and symptoms of acute KD have disappeared. Given that $\mathrm{KD}$ is a self-limited illness that resolves even in the absence of treatment, the diagnosis may be missed and the illness forgotten. Therefore, pediatrics and otolaryngologists should refer symptomatic patients for audiologic and vestibular evaluations and for $\mathrm{KD}$ evaluations, such as an echocardiogram.

\section{Acknowledgments}

This work was financially supported by Ministry of Science, ICT and Future Planning (MSIP) in Korean government and Korea Industrial Technology Association (KOITA) as a study on the programs to support collaborative research among industry, academia and research institutes.

\section{Conflicts of interest}

The authors have no financial conflicts of interest.

\section{REFERENCES}

1) Luca NJ, Yeung RS. Epidemiology and management of Kawasaki disease. Drugs 2012;72:1029-38.

2) Kato H, Koike S, Yokoyama T. Kawasaki disease: effect of treatment on coronary artery involvement. Pediatrics 1979;63:175-9.

3) Ichida F, Fatica NS, Engle MA, O’Loughlin JE, Klein AA, Snyder $\mathrm{MS}$, et al. Coronary artery involvement in Kawasaki syndrome in Manhattan, New York: risk factors and role of aspirin. Pediatrics 1987;80:828-35.

4) Sudo D, Monobe Y, Yashiro M, Sadakane A, Uehara R, Nakamura Y. Case-control study of giant coronary aneurysms due to Kawasaki disease: the 19th nationwide survey. Pediatr Int 2010;52:790-4.

5) Knott PD, Orloff LA, Harris JP, Novak RE, Burns JC; Kawasaki Disease Multicenter Hearing Loss Study Group. Sensorineural hearing loss and Kawasaki disease: a prospective study. Am J Otolaryngol 2001;22:343-8.

6) Novo A, Pinto S, Prior AC, Alvares S, Soares T, Guedes M. Kawasaki disease and sensorineural hearing loss: an (un)expected complication. Eur J Pediatr 2012;171:851-4.

7) Alves NR, Magalhães CM, Almeida Rde F, Santos RC, Gandolfi L, Pratesi R. Prospective study of Kawasaki disease complications: review of 115 cases. Rev Assoc Med Bras (1992) 2011;57:295-300.

8) Rowley AH, Shulman ST. New developments in the search for the etiologic agent of Kawasaki disease. Curr Opin Pediatr 2007;19:71-4.

9) Cimaz R, Falcini F. An update on Kawasaki disease. Autoimmun Rev 2003;2:258-63.

10) Harris JP. Immunologic mechanisms in disorders of the inner ear. In: Cummings CW, Krause CJ, Schuller DE, editors. Otolaryngology-Head and Neck Surgery. St. Louis, MO: CV Mosby Company; 1986.

11) Pagnoux C, Guillevin L. Peripheral neuropathy in systemic vasculitides. Curr Opin Rheumatol 2005; 17:41-8.

12) Brien JA. Ototoxicity associated with salicylates. A brief review. Drug Saf 1993;9:143-8.

13) Thornton AR, Farrell G. Apparent travelling wave velocity changes in cases of endolymphatic hydrops. Scand Audiol 1991;20:13-8.

14) Bushara K, Wilson A, Rust RS. Facial palsy in Kawasaki syndrome. Pediatr Neurol 1997;17:362-4. 